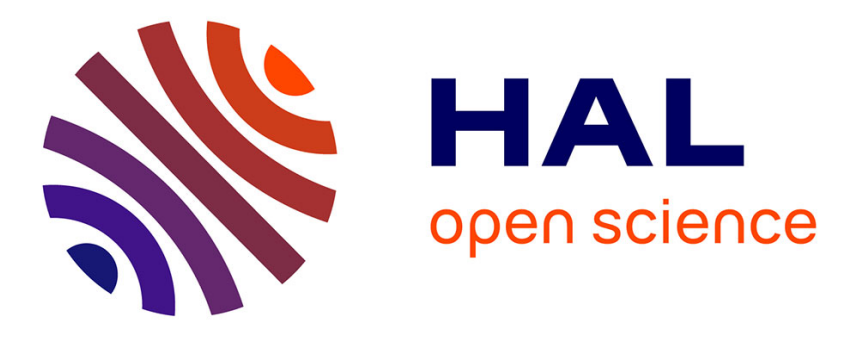

\title{
Factors affecting return to work after carpal tunnel syndrome surgery in a large French cohort
}

Elsa Parot-Schinkel, Yves Roquelaure, Catherine Ha, Annette Leclerc, Jean-François Chastang, Guy Raimbeau, Francis Chaise, Alexis Descatha

\section{- To cite this version:}

Elsa Parot-Schinkel, Yves Roquelaure, Catherine Ha, Annette Leclerc, Jean-François Chastang, et al.. Factors affecting return to work after carpal tunnel syndrome surgery in a large French cohort. Archives of Physical Medicine and Rehabilitation, 2011, 92 (11), pp.1863 - 1869. 10.1016/j.apmr.2011.06.001 . hal-03389889

\section{HAL Id: hal-03389889 \\ https://univ-angers.hal.science/hal-03389889}

Submitted on 21 Oct 2021

HAL is a multi-disciplinary open access archive for the deposit and dissemination of scientific research documents, whether they are published or not. The documents may come from teaching and research institutions in France or abroad, or from public or private research centers.
L'archive ouverte pluridisciplinaire HAL, est destinée au dépôt et à la diffusion de documents scientifiques de niveau recherche, publiés ou non, émanant des établissements d'enseignement et de recherche français ou étrangers, des laboratoires publics ou privés. 


\title{
Factors Affecting Return to Work After Carpal Tunnel Syndrome Surgery in a Large French Cohort
}

\author{
Elsa Parot-Schinkel, MD, Yves Roquelaure, MD, Catherine Ha, MD, Annette Leclerc, PhD, \\ Jean-François Chastang, PhD, Guy Raimbeau, MD, Francis Chaise, MD and Alexis Descatha, MD
}

ABSTRACT. Parot-Schinkel E, Roquelaure Y, Ha C, Leclerc A, Chastang J-F, Raimbeau G, Chaise F, Descatha A. Factors affecting return to work after carpal tunnel syndrome surgery in a large French cohort. Arch Phys Med Rehabil 2011; 92:1863-9.

Objective: To evaluate occupational outcomes after surgical release of the median nerve in carpal tunnel syndrome (CTS).

Design: Retrospective study 12 to 24 months after surgery.

Setting: Hand centers $(\mathrm{N}=3)$ in 2 different areas.

Participants: Patients who had undergone surgical release of the median nerve in 2002 to 2003.

Interventions: Not applicable.

Main Outcome Measure. Duration of sick leave after surgery and associated factors were analyzed by using bivariate (log rank) and multivariate analyses of survival (Cox model).

Results: Questionnaires mailed in 2004 regarding medical condition (history and surgery), employment (occupational category codes in 1 digit), and compensation were returned $(\mathrm{N}=1248 ; 62 \%)$, with 253 men and 682 women stating they were employed at the time of surgery $(\mathrm{N}=935)$. Most were working at the time of the study $(n=851 ; 91.0 \%)$. Median duration of sick leave before returning to work was 60 days. The main factors associated with adverse occupational outcome (long duration of sick leave) were simultaneous intervention for another upper-extremity musculoskeletal disorder, belief (by the patient) in an occupational cause, and "blue-collar worker" occupational category (the strongest determinant).

Conclusion: This study emphasizes the multifactorial nature of the occupational outcome of CTS after surgery, including occupational category. The probability of return to work for

From the Laboratory of Ergonomics and Epidemiology in Occupational Health, University of Angers, Angers (Parot-Schinkel, Roquelaure); Department of Occupational Health, French Institute for Public Health Surveillance (InVS), St-Maurice (Ha); INSERM U1018, Centre for Research in Epidemiology and Population Health, Epidemiology of Occupational and Social Determinants of Health, Villejuif (Leclerc, Chastang, Descatha); Université de Versailles St-Quentin, UMRS 1018 (Leclerc, Chastang, Descatha); the Hand Center, Angers (Raimbeau); Jeanne d'Arc Hospital, Nantes (Chaise); and AP-HP, Poincaré University Hospital, Occupational Health Department, Garches, France (Descatha).

Presented orally to PREMUS 2010, Seventh International Scientific Conference on Prevention of Work-Related Musculoskeletal Disorders, August 29-September 3, 2010, Angers, France.

Supported by the French Institute for Public Health Surveillance, Saint-Maurice, France (grant no. 9/25/2002-5 "réseau expérimental de surveillance des troubles musculo-squelettiques") and the French National Research Agency (ANR-grant no. SEST-06-36).

No commercial party having a direct financial interest in the results of the research supporting this article has or will confer a benefit on the authors or on any organization with which the authors are associated.

Reprints are not available from the author.

Correspondence to Alexis Descatha, MD, INSERM U1018, UVSQ, Unité de pathologie professionnelle U1018, CHU Poincaré, 104 bd Poincaré, 92380 Garches, France, e-mail: alexis.descatha@rpc.aphp.fr.

0003-9993/11/9211-00381\$36.00/0

doi:10.1016/j.apmr.2011.06.001 each risk factor provides a fair description of prognosis for physicians and patients.

Key Words: Carpal tunnel syndrome; Musculoskeletal disorders; Occupational disease; Prognosis; Rehabilitation; Return to work.

(C) 2011 by the American Congress of Rehabilitation Medicine

CARPAL TUNNEL syndrome (CTS) is the most common peripheral entrapment neuropathy in the upper extremity and a frequent cause of disability. ${ }^{1,2}$ Surgical release or decompression rates in the United States are 250,000 to 500,000 per year compared with 130,000 to 155,000 per year in France. ${ }^{3}$ Medical outcomes of surgical treatment of CTS have been studied in the last 20 years to evaluate preoperative predictors of prognosis, ${ }^{4-8}$ such as the potential effects of the surgical techniques. ${ }^{9-11}$ Return to work has been studied by using community-based studies and compensation-based studies, mostly in the United States. ${ }^{12-18}$

The aim of the study was to conduct a population-based investigation of surgical cases using hospital databases to define patterns of return to work in a general population and to analyze factors associated with return to work more than 1 year after surgery.

\section{METHODS}

A retrospective study involving subjects who had undergone surgery was conducted in 2004 in 2 French areas, the Maine and Loire area in 2002 and 2003 and the Loire-Atlantique area in 2003. The Pays de la Loire region was chosen to develop the French National surveillance system on CTS and other musculoskeletal disorders in 2002 because this region was representative of the French workforce. ${ }^{2,3}$ Subjects were included by using the National Medical Information Systems Program.

The search criteria were "carpal tunnel syndrome release" and "carpal tunnel syndrome ambulatory release" to include all patients aged 20 to 59 years living in these areas who had undergone carpal tunnel surgery. We included patients from only the main hand surgery centers $(75 \%$ of CTS surgery of the areas, 2002 data from the National Medical Information Systems Program), Centre de la Main (Maine \& Loire), Clinique St Léonard (Maine \& Loire), Clinique Jeanne d'Arc (Loire-Atlantique). Surgery teams have performed mini-open surgery for many years (under direct vision and minimally invasive technique with division of the transverse carpal ligament through a small and remote incision). ${ }^{19}$

Patients with incorrect mail addresses or already included in another study were excluded.

\section{List of Abbreviations}

CTS carpal tunnel syndrome

OCC occupation category code 


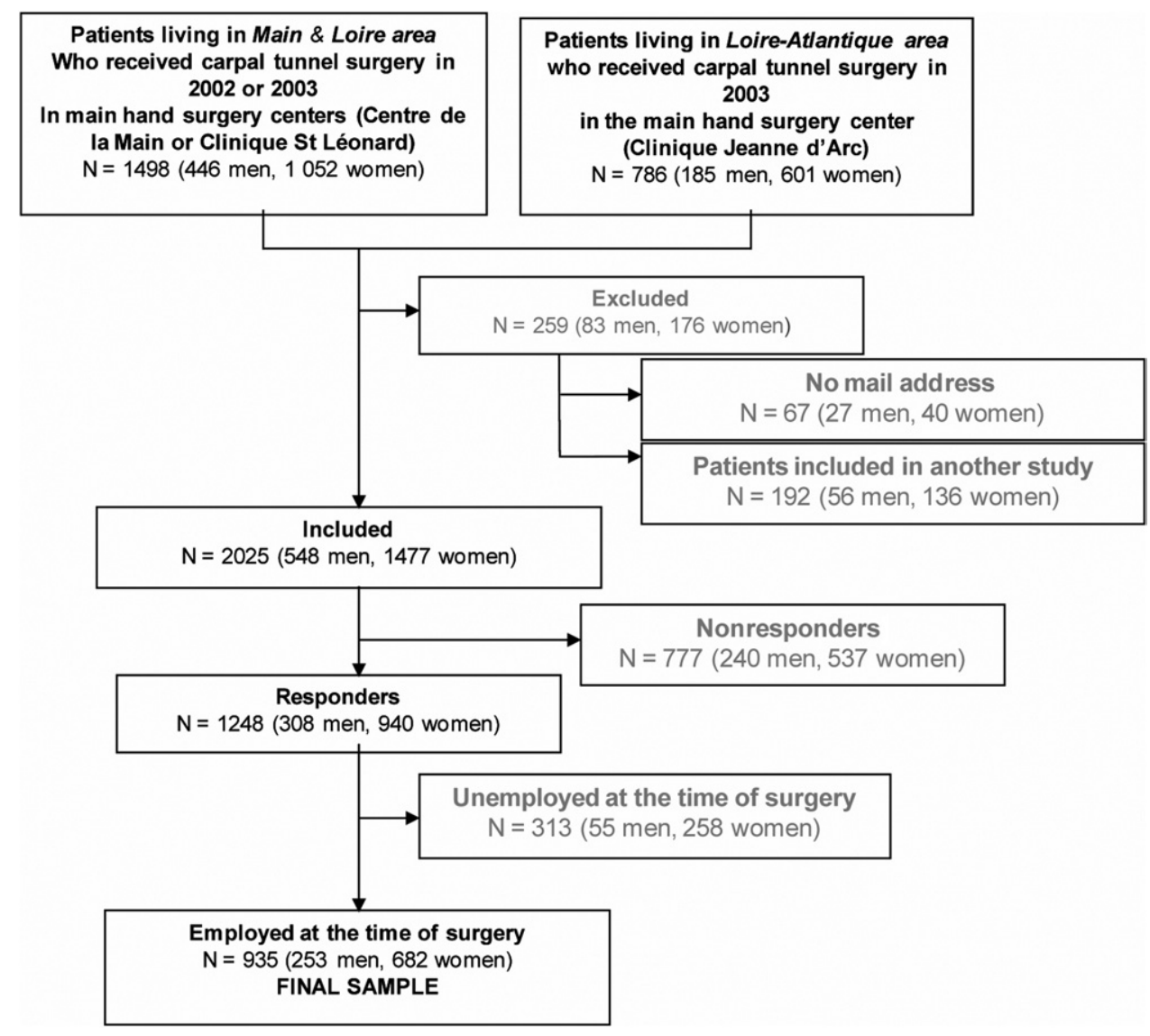

Fig 1. Flow diagram.

The final sample was based on responders to the questionnaire who were employed at the time of surgery (fig 1).

A self-administered questionnaire was mailed to each subject in 2004. Information was collected as follows. Medical history: obesity, pregnancy, diabetes mellitus, thyroid disease, upper-limb trauma (eg, fracture), rheumatoid arthritis, and any other upper-limb musculoskeletal disorder; surgery: uni- or bilateral release, associated surgery (at the same time), and satisfaction with results; more than a 2 -year interval between symptoms and surgery also was recorded; employment and job: the last job was coded according to the French classification of occupations (occupation category codes [OCC], 1 digit), over 15 years in the same job; and outcome: return to work and duration of sick leave, and modification of work task.

Information regarding sick leave payment under the workers' compensation system and the subjective imputation of causality by the patient also were requested (missing data were coded as no sickness pay under the workers' compensation system and no imputation of causality to work, respectively).

The main outcome was duration of sick leave after surgery, with the patient as the unit (not hand). Duration included the day of surgery to the end of sick leave, when the patient returned to any work (with or without restriction). In view of the retrospective design, the main occupational factor was the OCC. When patients had not recovered at the time of the questionnaire, they were considered censured and duration of sick leave was the time between surgery and answering the questionnaire.

After comparing responders and nonresponders to the questionnaire, the sample was analyzed. Subjects who returned to any work in 2004 were compared with those who did not (secondary outcome) by using bivariate and multivariate analyses (logistic model). Probability of return to work based on duration of sick leave was estimated by using the Kaplan-Meier estimator. Bivariate analyses using the log-rank test and multivariate analyses using the Cox model were performed including all relevant items. Pregnancy was not included in multivariate analyses. Stratification for sex and area was performed to check that results were valid for men and women and for the 2 geographic areas included in the study.

SAS, version $9.1,^{\mathrm{a}}$, was used for all statistical analyses. Associations were considered significant for $P<.05$.

\section{RESULTS}

Of 2025 subjects included, 1248 responded to the questionnaire $(61.6 \%$; see fig 1$)$.

Sex ratio and age were significantly different between responders and nonresponders in the Maine and Loire area (women/men, 3.1 vs $1.7 ; P<.001 ;$ age, $47 \pm 8$ vs $46 \pm 9 y$; $P=.033$, respectively). No difference was observed in the Loire-Atlantique area (sex ratio, 3.0 vs 3.7 women/men; age, $46 \pm 9$ vs $46 \pm 8 y$, respectively).

Of 1248 subjects in the responding groups, 935 were employed at the time of surgery and included in the final sample. Most were working at the time of the study $(n=851 ; 91.0 \%)$. Various factors were associated with the lack of return to work, including bilateral release, associated surgery, other musculoskeletal disorders, OCC, and dissatisfaction with surgery, whereas sickness payment under the workers' compensation system seemed protective (table 1). Stratified analyses indi- 
Table 1: Characteristics of Subjects Who Did Not Return to Work and Comparison With Subjects Who Returned to Work

\begin{tabular}{|c|c|c|c|c|c|c|c|c|}
\hline \multirow[b]{2}{*}{ Variable } & \multirow[b]{2}{*}{ Category } & \multirow[b]{2}{*}{ Total N } & \multicolumn{3}{|c|}{$\begin{array}{c}\text { Subjects Who Returned } \\
\text { to Work }\end{array}$} & \multicolumn{3}{|c|}{$\begin{array}{l}\text { Logistic Model }{ }^{*} \text { (reference }= \\
\text { return to work) }\end{array}$} \\
\hline & & & $\mathrm{n}$ & $\%$ & $P^{\dagger}$ & $\mathrm{aOR}$ & $95 \% \mathrm{Cl}$ & $P$ \\
\hline \multirow[t]{2}{*}{ Sex } & Women & 682 & 624 & 91.5 & NS & 1 & & NS \\
\hline & Men & 253 & 227 & 89.7 & & 0.95 & $0.47-1.89$ & \\
\hline \multirow[t]{2}{*}{ Area } & Loire Atlantique & 332 & 303 & 91.3 & NS & 1 & & NS \\
\hline & Maine et Loire & 603 & 548 & 90.9 & & 1.07 & $0.95-1.22$ & \\
\hline \multirow[t]{2}{*}{ Age $>50 y$} & No & 596 & 555 & 93.1 & .005 & 1 & & .02 \\
\hline & Yes & 336 & 294 & 87.5 & & 1.94 & $1.10-3.43$ & \\
\hline \multirow[t]{2}{*}{ Bilateral release } & No & 852 & 784 & 92 & .005 & 1 & & .003 \\
\hline & Yes & 81 & 65 & 80.3 & & 3.46 & $1.51-7.93$ & \\
\hline \multirow[t]{3}{*}{ Associated surgery } & No & 733 & 677 & 92.4 & .02 & 1 & & NS \\
\hline & Yes & 177 & 153 & 86.4 & & 1.47 & $0.78-2.80$ & \\
\hline & Missing data & 25 & 21 & 84 & & & & \\
\hline \multirow[t]{3}{*}{ Obesity $^{\ddagger}$} & No & 771 & 711 & 92.2 & .02 & 1 & & NS \\
\hline & Yes & 145 & 123 & 84.8 & & 1.88 & $0.95-3.73$ & \\
\hline & Missing data & 19 & 17 & 89.5 & & & & \\
\hline \multirow[t]{3}{*}{ Pregnancy } & No & 599 & 548 & 91.5 & NS & $\mathrm{NI}$ & & $\mathrm{NI}$ \\
\hline & Yes & 51 & 49 & 96.1 & & & & \\
\hline & Missing data + men & 285 & 254 & 89.1 & & & & \\
\hline \multirow[t]{3}{*}{ Diabetes mellitus } & No & 887 & 811 & 91.4 & NS & $\mathrm{NI}$ & & $\mathrm{NI}$ \\
\hline & Yes & 40 & 34 & 85 & & & & \\
\hline & Missing data & 8 & 6 & 75 & & & & \\
\hline \multirow[t]{3}{*}{ Thyroid disease } & No & 847 & 772 & 91.2 & NS & $\mathrm{NI}$ & & $\mathrm{NI}$ \\
\hline & Yes & 79 & 73 & 92.4 & & & & \\
\hline & Missing data & 9 & 6 & 66.7 & & & & \\
\hline \multirow[t]{2}{*}{ Upper-limb trauma (fracture) } & No & 899 & 819 & 91.1 & NS & $\mathrm{NI}$ & & $\mathrm{NI}$ \\
\hline & Yes & 36 & 32 & 88.9 & & & & \\
\hline \multirow[t]{2}{*}{ Rheumatoid arthritis } & No & 922 & 840 & 91.1 & NS & $\mathrm{NI}$ & & $\mathrm{NI}$ \\
\hline & Yes & 13 & 11 & 84.6 & & & & \\
\hline \multirow[t]{2}{*}{ Any personal risk factor for $\mathrm{CTS}^{\S}$} & No & 631 & 581 & 92.1 & NS & $\mathrm{NI}$ & & $\mathrm{NI}$ \\
\hline & At least 1 & 304 & 270 & 88.8 & & & & \\
\hline Other musculoskeletal disorders & No & 462 & 437 & 94.6 & .0001 & 1 & & .01 \\
\hline & Yes & 463 & 407 & 87.9 & & 2.18 & $1.21-3.95$ & \\
\hline & Missing data & 10 & 7 & 70 & & & & \\
\hline OCC & Farmers & 31 & 30 & 96.8 & .05 & - & & \\
\hline & Self-employed & 28 & 27 & 96.4 & & $\|_{1}$ & & .004 \\
\hline & Managers, executives & 42 & 42 & 100 & & - & & \\
\hline & Intermediates & 125 & 117 & 93.6 & & 3.77 & $0.71-20.01$ & \\
\hline & Lower white-collar workers & 353 & 319 & 90.4 & & 4.57 & $0.98-21.39$ & \\
\hline & Blue-collar workers & 356 & 316 & 88.8 & & 11.00 & $2.30-52.64$ & \\
\hline Time between first symptoms and surgery & $<2 y$ & 515 & 474 & 92 & NS & $\mathrm{NI}$ & & $\mathrm{NI}$ \\
\hline & $\geq 2 y$ & 420 & 377 & 89.8 & & & & \\
\hline Subjective imputation of cause to work & No & 76 & 75 & 98.7 & .051 & 1 & & NS \\
\hline & Yes & 839 & 758 & 90.4 & & 5.12 & $0.67-39.31$ & \\
\hline & Missing data & 20 & 18 & 90 & & & & \\
\hline$>15 y$ at the same job & No & 612 & 562 & 91.8 & NS & $\mathrm{NI}$ & & $\mathrm{NI}$ \\
\hline & Yes & 310 & 277 & 89.4 & & & & \\
\hline & Missing data & 13 & 12 & 92.3 & & & & \\
\hline Dissatisfaction with surgery & No & 772 & 717 & 92.9 & $<.0001$ & 1 & & .02 \\
\hline & Yes & 131 & 112 & 85.5 & & 2.22 & $1.12-4.40$ & \\
\hline & Missing data & 32 & 22 & 68.8 & & & & \\
\hline Sickness payment under the workers' & No & 583 & 504 & 86.5 & $<.0001$ & 1 & & .0001 \\
\hline & Yes & 352 & 347 & 98.6 & & 0.07 & $0.03-0.20$ & \\
\hline Modification of task & No & 819 & 737 & 90 & .004 & 1 & & NS \\
\hline & Yes & 116 & 114 & 98.3 & & 0.32 & $0.07-1.41$ & \\
\hline
\end{tabular}

NOTE. OR greater than 1 means that subjects were less likely to have returned to work.

Abbreviations: aOR, adjusted odds ratio; $\mathrm{Cl}$, confidence interval; NS, not significant $(P \geq .05), \mathrm{NI}=$ not included

* Multivariate analysis.

${ }^{\dagger}$ Univariate analysis.

${ }^{\ddagger}$ Body mass index $\geq 30 \mathrm{~kg} / \mathrm{m}^{2}$.

${ }^{\S}$ Obesity, pregnancy, diabetes mellitus, thyroid disease, upper-limb trauma, or rheumatoid arthritis.

"Farmers, self-employed, managers, and executives were merged together for the logistic model (reference group), taking into account that managers and executives always returned to work. 
Table 2: Probability of Return to Work in Days for Each Item (using Kaplan Meyer estimator) and Result of Log-Rank Test

\begin{tabular}{|c|c|c|c|c|c|c|c|c|}
\hline \multirow[b]{2}{*}{ Variable } & \multicolumn{7}{|c|}{ Probability of Return to Work $(95 \% \mathrm{Cl})$} & \multirow{2}{*}{$\underset{P}{\log -\operatorname{Rank}}$} \\
\hline & & $30 \mathrm{~d}$ & $60 \mathrm{~d}$ & $91 \mathrm{~d}$ & $182 \mathrm{~d}$ & $273 d$ & $364 d$ & \\
\hline \multirow[t]{2}{*}{ Sex } & Women & $19.0(16.2-22.2)$ & $58.3(54.6-62.0)$ & $76.4(73.2-79.6)$ & $87.5(84.9-89.8)$ & $90.5(88.1-92.5)$ & $91.1(88.8-93.1)$ & .04 \\
\hline & Men & $15.4(11.5-20.5)$ & $52.6(46.6-58.8)$ & $71.1(65.5-76.6)$ & $83.0(78.1-87.3)$ & $87.1(82.6-90.9)$ & $88.3(84.0-91.9)$ & \\
\hline \multirow[t]{2}{*}{ Area } & Loire Atlantique & $14.6(11.2-18.9)$ & $63.8(58.6-69.0)$ & $78.4(73.8-82.7)$ & $86.0(82.0-89.5)$ & $90.3(86.8-93.2)$ & $91.2(87.8-93.9)$ & .04 \\
\hline & Maine et Loire & $19.9(16.9-23.3)$ & $52.9(49.0-56.9)$ & $73.1(69.5-76.6)$ & $86.4(83.5-89.0)$ & $89.1(86.5-91.5)$ & $89.9(87.3-92.2)$ & \\
\hline \multirow[t]{2}{*}{ Age $>50 y$} & No & $14.7(12.1-17.8)$ & $55.8(51.9-59.8)$ & $75.7(72.2-79.1)$ & $87.9(85.1-90.3)$ & $91.6(89.2-93.6)$ & $92.5(90.1-94.4)$ & NS \\
\hline & Yes & $23.5(19.3-28.4)$ & $58.3(53.1-63.6)$ & $73.8(69.0-78.4)$ & $83.6(79.5-87.4)$ & $86.1(82.2-89.6)$ & $86.8(82.9-90.2)$ & \\
\hline \multirow[t]{2}{*}{ Bilateral release } & No & $18.2(15.8-21.0)$ & $57.8(54.5-61.1)$ & $76.7(73.8-79.5)$ & $87.4(85.1-89.5)$ & $90.6(88.6-92.5)$ & $91.3(89.3-93.1)$ & .0007 \\
\hline & Yes & $16.2(9.8-26.3)$ & $46.2(36.1-57.7)$ & $56.2(45.8-67.3)$ & $73.7(63.8-82.8)$ & $77.5(67.9-85.9)$ & $80.0(70.6-87.9)$ & \\
\hline \multirow[t]{2}{*}{ Associated surgery } & No & $18.9(16.2-21.9)$ & $61.0(57.5-64.6)$ & $80.0(77.1-82.8)$ & $89.2(86.8-91.3)$ & $91.9(89.7-93.7)$ & $92.6(90.6-94.4)$ & .0000 \\
\hline & Yes & $13.6(9.4-19.7)$ & $39.2(32.4-46.8)$ & $56.3(49.1-63.7)$ & $75.0(68.4-81.1)$ & $80.7(74.5-86.1)$ & $81.9(75.8-87.2)$ & \\
\hline \multirow[t]{2}{*}{ Obesity* } & No & $18.5(15.9-21.4)$ & $58.4(54.9-61.9)$ & $76.2(73.1-79.2)$ & $87.3(84.8-89.5)$ & $90.7(88.5-92.6)$ & $91.4(89.3-93.3)$ & .0025 \\
\hline & Yes & $13.2(8.6-19.9)$ & $47.9(40.1-56.4)$ & $68.7(61.1-76.1)$ & $81.2(74.5-87.1)$ & $84.0(77.6-89.5)$ & $84.8(78.4-90.1)$ & \\
\hline \multirow[t]{2}{*}{ Pregnancy } & No & $19.0(16.0-22.3)$ & $57.7(53.8-61.7)$ & $76.2(72.7-79.5)$ & $87.2(84.4-89.8)$ & $90.3(87.8-92.5)$ & $91.1(88.6-93.2)$ & .0065 \\
\hline & Yes & $23.5(14.1-37.7)$ & $78.4(66.4-88.4)$ & $92.2(82.8-97.5)$ & $96.1(88.1-99.3)$ & $96.1(88.1-99.3)$ & $96.1(88.1-99.3)$ & \\
\hline \multirow[t]{2}{*}{ Diabetes mellitus } & No & $18.6(16.1-21.3)$ & $57.4(54.1-60.6)$ & $75.1(72.2-77.9)$ & $86.9(84.6-89.0)$ & $90.0(87.9-91.9)$ & $90.9(88.8-92.7)$ & NS \\
\hline & Yes & $10.0(3.9-24.5)$ & $47.5(33.5-63.9)$ & $80.0(66.5-90.6)$ & $80.0(66.5-90.6)$ & $82.5(69.4-92.3)$ & $82.5(69.4-92.3)$ & \\
\hline \multirow{2}{*}{ Thyroid disease } & No & $18.0(15.6-20.7)$ & $56.7(53.4-60.0)$ & $75.4(72.4-78.2)$ & $86.3(83.9-88.5)$ & $89.5(87.3-91.5)$ & $90.3(88.2-92.2)$ & NS \\
\hline & Yes & $20.5(13.1-31.3)$ & $60.3(49.7-71.1)$ & $75.6(65.7-84.5)$ & $91.0(83.4-96.1)$ & $92.3(85.1-96.9)$ & $93.8(86.9-97.8)$ & \\
\hline \multirow[t]{2}{*}{ Upper-limb trauma (fracture) } & No & $18.1(15.7-20.8)$ & $56.7(53.5-60.0)$ & $74.8(71.9-77.6)$ & $86.4(84.0-88.5)$ & $89.7(87.6-91.6)$ & $90.6(88.5-92.4)$ & NS \\
\hline & Yes & $16.7(7.9-33.4)$ & $58.3(43.0-74.4)$ & $80.6(66.4-91.4)$ & $83.3(69.6-93.2)$ & $86.1(72.9-94.9)$ & $86.1(72.9-94.9)$ & \\
\hline \multirow{2}{*}{ Rheumatoid arthritis } & No & $15.9(12.9-19.4)$ & $57.3(53.0-61.7)$ & $75.6(71.7-79.3)$ & $86.6(83.4-89.4)$ & $90.7(87.9-93.0)$ & $91.5(88.8-93.7)$ & NS \\
\hline & Yes & $46.2(24.0-75.2)$ & $69.2(44.6-90.5)$ & $84.6(61.2-97.5)$ & $84.6(61.2-97.5)$ & $84.6(61.2-97.5)$ & $84.6(61.2-97.5)$ & \\
\hline \multirow[t]{2}{*}{ Any personal risk factor for $\mathrm{CTS}^{\dagger}$} & No & $18.4(15.6-21.7)$ & $56.9(53.1-60.8)$ & $75.2(71.8-78.5)$ & $86.8(84.0-89.3)$ & $90.5(88.1-92.7)$ & $91.5(89.1-93.5)$ & NS \\
\hline & At least 1 & $17.2(13.4-21.9)$ & $56.4(50.9-62.1)$ & $74.6(69.6-79.3)$ & $85.1(80.9-88.9)$ & $87.5(83.5-90.9)$ & $88.1(84.2-91.5)$ & \\
\hline \multirow{2}{*}{ Other musculoskeletal disorders } & No & $19.6(16.2-23.5)$ & $62.8(58.4-67.2)$ & $80.4(76.7-83.9)$ & $90.9(88.0-93.3)$ & $94.2(91.8-96.1)$ & $94.8(92.5-96.6)$ & .0000 \\
\hline & Yes & $16.9(13.8-20.6)$ & $51.1(46.6-55.7)$ & $70.3(66.1-74.4)$ & $82.3(78.6-85.6)$ & $85.3(81.9-88.4)$ & $86.3(83.0-89.3)$ & \\
\hline \multirow[t]{6}{*}{ OCC } & Farmers & $51.6(35.6-69.8)$ & $80.6(65.4-92.1)$ & $87.1(73.0-95.9)$ & $96.8(85.9-99.8)$ & $96.8(85.9-99.8)$ & $96.8(85.9-99.8)$ & .0000 \\
\hline & Self-employed & $64.3(47.0-81.1)$ & $89.3(74.9-97.3)$ & $92.9(79.6-98.7)$ & $96.4(84.6-99.7)$ & $96.4(84.6-99.7)$ & $96.4(84.6-99.7)$ & \\
\hline & Managers, executives & $65.9(51.5-79.7)$ & $92.7(82.2-98.1)$ & $97.6(89.0-99.8)$ & $97.6(89.0-99.8)$ & 100 & 100 & \\
\hline & Intermediates & $22.4(16.0-30.8)$ & $72.0(64.0-79.6)$ & $84.8(78.0-90.4)$ & $91.2(85.4-95.3)$ & $93.6(88.4-97.0)$ & $93.6(88.4-97.0)$ & \\
\hline & Lower white-collar workers & $15.7(12.3-19.9)$ & $59.8(54.7-65.0)$ & $79.5(75.1-83.5)$ & $88.6(85.0-91.7)$ & $89.8(86.3-92.7)$ & $90.4(87.0-93.2)$ & \\
\hline & Blue-collar workers & $6.5(4.4-9.6)$ & $39.4(34.6-44.7)$ & $62.0(56.9-67.0)$ & $79.2(74.8-83.2)$ & $85.5(81.6-88.9)$ & $87.0(83.2-90.2)$ & \\
\hline \multirow{2}{*}{$\begin{array}{l}\text { Time between first symptoms } \\
\text { and surgery }\end{array}$} & $<2 y$ & $15.6(12.7-19.0)$ & $56.7(52.5-61.0)$ & $77.4(73.7-80.9)$ & $87.9(84.9-90.6)$ & $91.1(88.4-93.3)$ & $91.7(89.1-93.9)$ & NS \\
\hline & $\geq 2 y$ & $21.0(17.4-25.2)$ & $56.8(52.1-61.6)$ & $72.1(67.7-76.3)$ & $84.2(80.6-87.6)$ & $87.7(84.3-90.6)$ & $88.7(85.4-91.5)$ & \\
\hline \multirow{2}{*}{$\begin{array}{l}\text { Subjective imputation of cause } \\
\text { to work }\end{array}$} & No & $57.9(47.2-69.1)$ & $85.5(76.7-92.3)$ & $94.7(88.1-98.3)$ & $97.4(91.8-99.5)$ & $97.4(91.8-99.5)$ & 100 & .0000 \\
\hline & Yes & $14.0(11.8-16.5)$ & $53.6(50.2-57.0)$ & $73.0(69.9-75.9)$ & $85.2(82.7-87.5)$ & $88.8(86.6-90.8)$ & $89.6(87.4-91.6)$ & \\
\hline \multirow[t]{2}{*}{$>15 y$ at the same job } & No & $18.1(15.2-21.4)$ & $58.5(54.6-62.4)$ & $77.3(73.9-80.6)$ & $87.7(84.9-90.1)$ & $90.6(88.2-92.8)$ & $91.5(89.1-93.6)$ & .0455 \\
\hline & Yes & $17.7(13.9-22.5)$ & $52.9(47.5-58.6)$ & $70.0(64.8-75.0)$ & $83.2(78.9-87.1)$ & $87.3(83.3-90.7)$ & $88.0(84.1-91.4)$ & \\
\hline \multirow[t]{2}{*}{ Dissatisfaction with surgery } & No & $18.7(16.1-21.6)$ & $60.2(56.7-63.6)$ & $78.1(75.1-80.9)$ & $89.4(87.1-91.4)$ & $91.7(89.6-93.5)$ & $92.3(90.3-94.0)$ & . 0001 \\
\hline & Yes & $15.4(10.2-22.8)$ & $42.3(34.4-51.3)$ & $62.3(54.1-70.6)$ & $73.1(65.3-80.4)$ & $81.8(74.7-87.9)$ & $83.5(76.5-89.3)$ & \\
\hline & No & $24.5(21.2-28.2)$ & $67.9(64.1-71.7)$ & $80.3(77.0-83.5)$ & $84.8(81.8-87.6)$ & $86.1(83.1-88.7)$ & $86.4(83.5-89.1)$ & . 0089 \\
\hline & Yes & $7.4(5.1-10.7)$ & $38.4(33.5-43.6)$ & $66.2(61.2-71.1)$ & $88.6(85.1-91.7)$ & $95.2(92.6-97.1)$ & $96.7(94.4-98.2)$ & \\
\hline Modification of task & No & $18.8(16.2-21.6)$ & $59.1(55.7-62.5)$ & $76.6(73.6-79.4)$ & $86.3(83.8-88.5)$ & $89.1(86.9-91.2)$ & $89.8(87.6-91.8)$ & NS \\
\hline & Yes & $12.9(8.0-20.5)$ & $40.5(32.2-50.0)$ & $63.8(55.1-72.4)$ & $86.2(79.3-91.7)$ & $92.2(86.5-96.2)$ & $94.0(88.6-97.3)$ & \\
\hline Total & & $18.0(15.7-20.6)$ & $56.8(53.6-60.0)$ & $75.0(72.2-77.7)$ & $86.3(84.0-88.4)$ & $89.5(87.5-91.4)$ & $90.4(88.4-92.2)$ & \\
\hline
\end{tabular}

Abbreviations: $\mathrm{Cl}$, confidence interval; NS, not significant $(P \geq .05)$.

*Body mass index $\geq 30 \mathrm{~kg} / \mathrm{m}^{2}$.

${ }^{\dagger}$ Obesity, pregnancy, diabetes mellitus, thyroid disease, upper-limb trauma, or rheumatoid arthritis. 
Fig 2. Survival-like curves using Kaplan-Meier estimator in each occupational category. $x$-axis, time between surgery and return to work; $y$-axis, percentage of return to work.
Log-Rank $p=.0000$

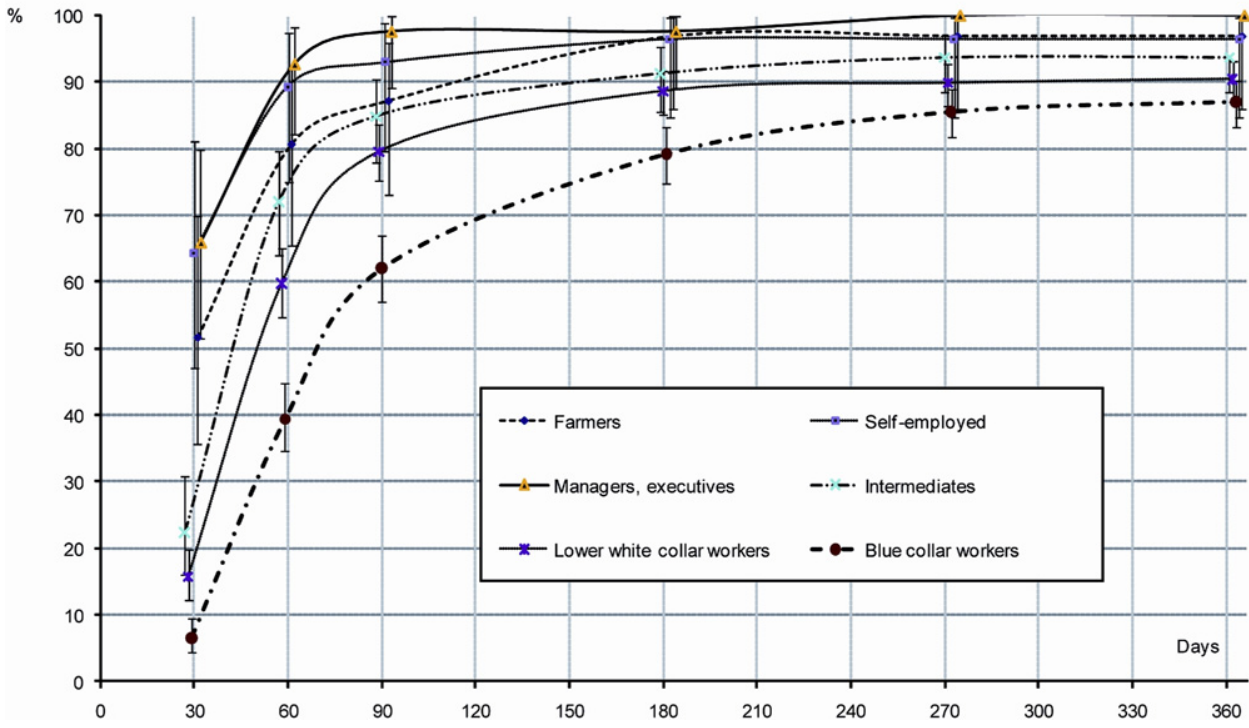

cated that results were similar for men and women and for the 2 areas.

Median duration of sick leave before return to work was 60 days. Different factors were associated with duration of sick leave before return to work in bivariate analyses (table 2): sex, area, bilateral release, other associated surgery, obesity, pregnancy, other upper-limb musculoskeletal disorder, OCC, subjective imputation of cause to work, time in the current job, dissatisfaction with surgery, and sickness payment under the workers' compensation system. OCC was the variable associated most strongly with duration of sick leave (fig 2). Other upper-limb musculosk- eletal disorders and work compensation were associated with OCC $(P<.05)$. However, after multivariate analyses by using a Cox model (table 3), only bilateral release, other associated surgery, obesity, other upper-limb musculoskeletal disorder, OCC, subjective imputation of cause to work, and dissatisfaction with surgery were still associated significantly with duration of sick leave. No difference between the 2 areas was observed after adjustment for factors associated with return to work (or between hand centers), such as sickness leave payment under the workers' compensation system.

Table 3: Results of Multivariate Analysis on Duration of Sick Leave (Cox model)

\begin{tabular}{|c|c|c|c|}
\hline Variable & Category & Hazard Ratio & $\operatorname{Pr}>\chi^{2}$ \\
\hline Sex & Men vs women & $0.98(0.82-1.18)$ & NS \\
\hline Area & Maine et Loire vs Loire Atlantique & $0.90(0.77-1.06)$ & NS \\
\hline Age $>50 y$ & Yes vs no & $1.03(0.87-1.20)$ & NS \\
\hline Bilateral release & Yes vs no & $1.41(1.05-1.87)^{\dagger}$ & .02 \\
\hline Associated surgery & Yes vs no & $1.37(1.13-1.67)^{\dagger}$ & .0015 \\
\hline Obesity* & Yes vs no & $1.25(1.02-1.54)^{\dagger}$ & NS \\
\hline Diabetes mellitus & Yes vs no & $1.22(0.82-1.83)$ & NS \\
\hline Upper-limb trauma (fracture) & Yes vs no & $1.17(0.79-1.72)$ & NS \\
\hline Rheumatoid arthritis & Yes vs no & $0.79(0.42-1.49)$ & NS \\
\hline Other musculoskeletal disorders & Yes vs no & $1.19(1.02-1.38)^{\dagger}$ & NS \\
\hline \multirow[t]{5}{*}{ OCC (reference: managers and executives) } & Farmers & $1.47(0.88-2.46)$ & $<.0001$ \\
\hline & Self employed & $1.08(0.63-1.86)$ & \\
\hline & Intermediates & $2.21(1.49-3.27)^{\dagger}$ & \\
\hline & Lower white-collar workers & $2.49(1.71-3.61)^{\dagger}$ & \\
\hline & Blue-collar workers & $3.34(2.28-4.90)^{\dagger}$ & \\
\hline Time between first symptoms and surgery & $\geq 2$ vs $<2 y$ & $1.01(0.87-1.16)$ & NS \\
\hline Subjective imputation of cause to work & Yes vs no & $1.88(1.43-2.48)^{\dagger}$ & $<.0001$ \\
\hline$>15 y$ at the same job & Yes vs no & $1.14(0.98-1.34)$ & NS \\
\hline Dissatisfaction with surgery & Yes vs no & $1.37(1.10-1.70)^{\dagger}$ & .0046 \\
\hline $\begin{array}{l}\text { Sickness payment under the workers' } \\
\text { compensation system }\end{array}$ & Yes vs no & $1.06(0.90-1.24)$ & NS \\
\hline Modification of the task & Yes vs no & $0.99(0.79-1.24)$ & NS \\
\hline
\end{tabular}

Abbreviations: $\mathrm{Cl}$, confidence interval; $\mathrm{NS}$, not significant $(P \geq .05)$.

*Body mass index $\geq 30 \mathrm{~kg} / \mathrm{m}^{2}$.

$+P<.05$. 


\section{DISCUSSION}

Most patients with CTS returned to work after surgery. However, certain factors were associated with duration of sick leave, and the average probability of return to work related to each of these factors provides a fair description of prognosis for physicians and patients. This is the first time that this description has been obtained and it will help clinicians inform their patients. The prognostic factors are multiple, including medical (obesity, other musculoskeletal disorders), surgical (bilateral release at the same time, associated surgery, satisfaction with surgery), and occupational parameters (OCC, subjective imputation of cause to work).

Because patients were drawn from 3 hand centers, it is possible that there might have been selection of patients. However, although located in the Pays-de-la-Loire region, these 3 major hand centers are not based in similar areas and they have small differences in surgical practice (associated surgery). No significant differences in outcome were observed with or without adjustment for a possible area effect (although external validation is required). Multilevel analyses were not performed in view of the lack of area effect. Mini-open surgery has been performed by the surgical teams for many years. Although the decision to apply endoscopic carpal tunnel release instead of open carpal tunnel release remains a matter of debate, it seems that it should be guided by the surgeon's preference. ${ }^{11,19-21}$

Occupational category appeared to be the strongest predictor of return to work and duration of sick leave, as reported in previous studies. In a study based on subjects who underwent surgery, Daniell et $\mathrm{al}^{22}$ found that sick leave was less likely to end before 6 months in industries with a high incidence of CTS. Exposure to intensive hand work also has been described as a poor predictor of return to work, ${ }^{12}$ at least in bivariate analysis at 6 months. ${ }^{16}$ Repetitive movement at work and heavy manual work were associated with a longer return to work interval in a cohort of surgical cases ${ }^{18}$ (without influence of occupational category, but with inclusion of either employees or self-employed) and also in a French study by a different team. $^{14}$

The relationship between sickness payment under a workers' compensation system and return to work is complex. Different results have been reported regarding the influence of workers' compensation. 7,17,23-25 Workers' compensation in some systems may help workers obtain better care and work improvement, as in France, but may lead to longer duration of sick leave before return to work. ${ }^{14}$ Workers' compensation also is potentially representative of work exposure and social factors (personal communication: G. Pransky, Prognosis for return to work in carpal tunnel syndrome: a North American perspective, Premus, 2010). ${ }^{25}$ It also may be a proxy for severity, bearing in mind that there is no major difference in France between the workers' compensation system and the usual heath care system, and patients with mild CTS usually do not ask for compensation. We found a strong association in this study between sickness payment under the workers' compensation system and OCC, but the self-reported workers' compensation and missing information led us to consider this item in our study with caution.

\section{Study Limitations}

However, this retrospective study was based on hospital data and such a design can lead to potential selection and recall effects. For occupational exposure, we chose to use OCC instead of self-reported exposure to avoid recall effects regarding exposure, but we were not able to distinguish which types of social or specific work exposure were the most relevant. It is true that OCC mixed different levels of physical demands (especially when they are pooled together for statistical reasons). Other baseline conditions, such as severity or intensity of pain at baseline and psychological variables, were not included in view of the possible memory effect associated with the retrospective design.

A potential attrition effect might have led to underestimation of the duration of sick leave ( $39.4 \%$ lost to follow-up). However, the average duration of sick leave reported in other studies has been 17 to 108 days, mostly depending on work characteristics and social categories (personal communication: G. Pransky, Prognosis for return to work in carpal tunnel syndrome: a North American perspective, Premus, 2010), 7,14,26 surgical treatment, ${ }^{22}$ and receipt of sick pay. ${ }^{14}$ The median of 60 days and proportion of return to work at 90 and 180 days are consistent with previous studies of similar design in unselected populations of workers. ${ }^{22,26-28}$ It is interesting that average duration is similar between these North American studies undertaken in a mainly independent labor system and our results in a protective labor system in which, for example, workers cannot be fired when they are on sick leave for this reason. The financial loss for workers during sickness leave also is minimal, at least for the first 3 months. In this protective system, it also is of note that patients who did not return to the previous job remained unemployed. The variable return to work usually means return to any work.

\section{CONCLUSION}

The association with other musculoskeletal disorders that may need surgery (associated surgery, bilateral release) or not (other musculoskeletal disorders) also is consistent with previous studies. ${ }^{16,29}$ This could be considered another obstacle to return to work and also as an indicator of a physically demanding job in cases of upper-limb tendonitis. An unfavorable work environment, including physical and psychosocial factors, ${ }^{26,30}$ often is considered to indicate poor prognosis, probably also in combination with disorders other than CTS. Dissatisfaction with surgery potentially is an interesting factor, but was difficult to interpret considering that it included expectations and results. However, for the role of workers' compensation and OCC, further prospective studies are needed to clarify which factors best predict return to work, including work exposure, severity at baseline, and use of a functional index.

\section{References}

1. Atroshi I, Gummesson C, Johnsson R, Ornstein E, Ranstam J, Rosen I. Prevalence of carpal tunnel syndrome in a general population. JAMA 1999;282:153-8.

2. Roquelaure Y, Ha C, Nicolas G, et al. Attributable risk of carpal tunnel syndrome according to industry and occupation in a general population. Arthritis Rheum 2008;59:1341-8.

3. Roquelaure Y, Ha C, Pelier-Cady MC, et al. Work increases the incidence of carpal tunnel syndrome in the general population. Muscle Nerve 2008;37:477-82.

4. Adams ML, Franklin GM, Barnhart S. Outcome of carpal tunnel surgery in Washington State workers' compensation. Am J Ind Med 1994;25:527-36.

5. Feuerstein M, Burrell LM, Miller VI, Lincoln A, Huang GD, Berger R. Clinical management of carpal tunnel syndrome: a 12-year review of outcomes. Am J Ind Med 1999;35:232-45.

6. Katz JN, Losina E, Amick BC III, Fossel AH, Bessette L, Keller RB. Predictors of outcomes of carpal tunnel release. Arthritis Rheum 2001;44:1184-93.

7. Schmelzer RE, Della Rocca GJ, Caplin DA. Endoscopic carpal tunnel release: a review of 753 cases in 486 patients. Plast Reconstr Surg 2006;117:177-85. 
8. Gardner BT, Dale AM, Vandillen L, Franzblau A, Evanoff BA. Predictors of upper extremity symptoms and functional impairment among workers employed for 6 months in a new job. Am J Ind Med 2008;51:932-40.

9. Gerritsen AA, Uitdehaag BM, van Geldere D, Scholten RJ, de Vet HC, Bouter LM. Systematic review of randomized clinical trials of surgical treatment for carpal tunnel syndrome. Br J Surg 2001; $88: 1285-95$

10. Atroshi I, Larsson GU, Ornstein E, Hofer M, Johnsson R, Ranstam J. Outcomes of endoscopic surgery compared with open surgery for carpal tunnel syndrome among employed patients: randomised controlled trial. BMJ 2006;332:1473, 1-8.

11. Scholten RJ, Mink vdM, Uitdehaag BM, Bouter LM, de Vet HC. Surgical treatment options for carpal tunnel syndrome. Cochrane Database Syst Rev 2007;CD003905.

12. Katz JN, Keller RB, Fossel AH, et al. Predictors of return to work following carpal tunnel release. Am J Ind Med 1997;31:85-91.

13. Carmona L, Faucett J, Blanc PD, Yelin E. Predictors of rate of return to work after surgery for carpal tunnel syndrome. Arthritis Care Res 1998;11:298-305.

14. Chaise F, Bellemere P, Fril JP, Gaisne E, Poirier P, Menadi A. Return-to-work interval and surgery for carpal tunnel syndrome. Results of a prospective series of 233 patients. J Hand Surg [Br] 2004;29:568-70.

15. Amick BC III, Habeck RV, Ossmann J, Fossel AH, Keller R, Katz JN. Predictors of successful work role functioning after carpal tunnel release surgery. J Occup Environ Med 2004;46:490-500.

16. Katz JN, Amick BC III, Keller R, et al. Determinants of work absence following surgery for carpal tunnel syndrome. Am J Ind Med 2005;47:120-30.

17. Ellis RA, Novak CB, Mackinnon SE, Cheng CJ. Workers' compensation, return to work, and patient satisfaction after carpal tunnel decompression. Am J Orthop 2007;36:E63-6.

18. De Kesel R, Donceel P, De Smet L. Factors influencing return to work after surgical treatment for carpal tunnel syndrome. Occup Med (Lond) 2008;58:187-90.

19. Sanati KA, Mansouri M, Macdonald D, Ghafghazi S, Macdonald E, Yadegarfar G. Surgical techniques and return to work following carpal tunnel release: a systematic review and meta-analysis. J Occup Rehabil 2011. April 29, 2011 [E-pub ahead of print].
20. Scholten RJ, Gerritsen AA, Uitdehaag BM, van Geldere D, de Vet HC, Bouter LM. Surgical treatment options for carpal tunnel syndrome. Cochrane Database Syst Rev 2002;CD003905.

21. Scholten RJ, Gerritsen AA, Uitdehaag BM, van Geldere D, de Vet HC, Bouter LM. Surgical treatment options for carpal tunnel syndrome. Cochrane Database Syst Rev 2004;CD003905.

22. Daniell WE, Fulton-Kehoe D, Franklin GM. Work-related carpal tunnel syndrome in Washington State workers' compensation: utilization of surgery and the duration of lost work. Am J Ind Med 2009;52:931-42.

23. Higgs PE, Edwards D, Martin DS, Weeks PM. Carpal tunnel surgery outcomes in workers: effect of workers' compensation status. J Hand Surg [Am] 1995;20:354-60.

24. Katz JN, Lew RA, Bessette L, et al. Prevalence and predictors of long-term work disability due to carpal tunnel syndrome. Am J Ind Med 1998;33:543-50.

25. Bitar G, Alexandrides J, Missirian R, Sotereanos D, Nystrom A. Carpal tunnel release in the United States and Sweden: reimbursement patterns, cost for treatment, and return to work. Plast Reconstr Surg 2002;109:1574-8.

26. Gimeno D, Amick BC III, Habeck RV, Ossmann J, Katz JN. The role of job strain on return to work after carpal tunnel surgery. Occup Environ Med 2005;62:778-85.

27. Pomerance J, Fine I. Outcomes of carpal tunnel surgery with and without supervised postoperative therapy. J Hand Surg [Am] 2007;32:1159-63.

28. Katz JN, Punnett L, Simmons BP, Fossel AH, Mooney N, Keller RB. Workers' compensation recipients with carpal tunnel syndrome: the validity of self-reported health measures. Am J Public Health 1996;86:52-6.

29. Manktelow RT, Binhammer P, Tomat LR, Bril V, Szalai JP. Carpal tunnel syndrome: cross-sectional and outcome study in Ontario workers. J Hand Surg [Am] 2004;29:307-17.

30. Silverstein BA, Fan ZJ, Bonauto DK, et al. The natural course of carpal tunnel syndrome in a working population. Scand J Work Environ Health 2010;36:384-93.

\section{Supplier}

a. SAS Institute Inc, 100 SAS Campus Dr, Cary, NC 27513-2414. 